\title{
Research on Milk Conductivity Real-time Online Monitoring System
}

\author{
Weizheng Shen, Wenxiao Yu, Qingming Kong, Yu Zhang, Guanting Liu \\ and Qi Wang \\ School of Electrical and Information \\ Northeast Agricultural University, Harbin, 150030, China \\ wzshen@neau.edu.cn
}

\begin{abstract}
Bovine mastitis is one of the common diseases in the dairy industry, and is also caused by the loss in the dairy industry. Timely and effective detection of bovine mastitis has important significance. By monitoring the milk conductivity values and other parameters, is to automate the monitoring cow health, is the basis of the prevention of bovine mastitis infection. This design is based single-chip microcomputer processing core control sensor measurement unit, data transmission unit, display and control unit, online monitoring system to achieve real-time data collection, transmission, display and other control functions. For monitoring the conductivity values were piecewise linear model of the relationship between the conductivity and the feedback voltage power function curve model comparison with simulation experiment. Simulation results show that the conductivity measurement piecewise linear model is a better, fitter factor, the average relative error is $2.31 \%$ lower, measuring range is $0.7 \mathrm{~ms} / \mathrm{cm}-11.7 \mathrm{~ms} / \mathrm{cm}$. The error of measuring the overall system is of relative low ratio, but the accuracy is higher, and better meets the needs of real-time monitoring, for cow health management and prevention of bovine mastitis or other diseases provide effective technical means.
\end{abstract}

Keywords: Bovine Mastitis, Conductivity, Real-time Online Monitoring, Curve fitting, simulation experiment

\section{Introduction}

Bovine mastitis is an infection caused by pathogenic microorganisms is a complex inflammatory disease of the breast, is one of the major diseases hazards of dairy industry development. Numerous factors cause mastitis in dairy cows, such as: environmental management factors, infections and other pathogens[1-3]. The most effective way to control and prevention of this disease it is effective for rapid detection.

Currently, there are many methods to detect mastitis in dairy cows, somatic cell count, chemical test method, but there are detected long period, high cost, and low degree of automation shortcomings. Given this situation, we sick cow's milk according to changes in that occur in the physical and chemical properties and bacteriology, conduct research on the electrical conductivity of milk, milk temperature, milk and other parameters of the measured value analysis, the results show that the online monitoring of these parameters can detect cows suffering from mastitis, The results show that the online monitoring of these parameters can detect cows suffering from mastitis, sick cows have been timely and effective treatment, and help prevent and control cows other breast diseases, help to extend cow milk production cycle, improve its annual production of milk[4-6].At home and abroad have developed a variety of conductivity measurement instruments, but because the farm milking parlor environment and other factors limit, and it cannot meet the requirements of real-time monitoring[7-9]. The objective of this study is to develop a real-time online monitoring system for milk conductivity, change the traditional milk 
conductivity monitoring mode, for the prevention of mastitis in dairy cows disease provide effective technical means, improve the intelligence level of the milk conductivity measurement, achieve a milk conductivity monitoring automation management.

\section{Monitoring System Development Description}

\subsection{The Overall Design of the System Description}

This system research, mainly through the use of single-chip microcomputer with sensors to achieve the data collection, online display, and through data transmission unit will upload the data to the host computer, to achieve real-time monitoring of milk conductivity. At the same time, taking into account the needs of the practical application of the system, the system can be set conductivity detection reference point for real-time monitoring of udder health.

Figure 1 is the overall design of the monitoring system, the monitoring system includes several components such as measurements, transmission, PC server and so on.

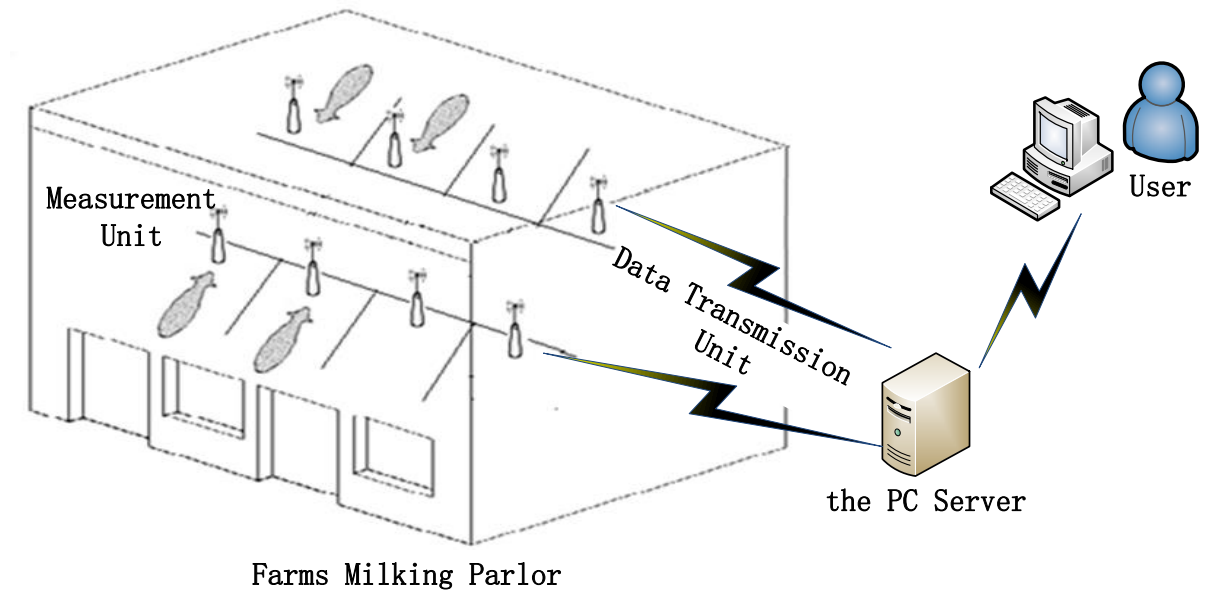

Figure 1. The Overall Design of the Monitoring System

Measuring unit includes a single-chip microprocessor core with sensors for collecting and displaying the cumulative milking time, milk conductivity, temperature and other data. Measuring unit is mainly placed in farms milking parlor, a measurement node is installed on each milking position. The actual installation location is between automatic milking machines and milk collection container. Data measurement unit collected data can be uploaded via the transmission unit to a PC server. It's the specific number and placement can be adjusted according to the actual situation. Data transmission unit mainly uses RS485 communication mode, is responsible for measuring data transmission between the unit and PC servers, is about measuring unit collected data accurate information in real time via the communication interface to upload to the PC server side. Data are then displayed to the user through the processing, improves the overall usability of the system.

\subsection{Hardware Design of the System Description}

Figure 2 is a hardware structure diagram of the measurement and the transmission unit. It includes Single Chip Microprocessor, LCD display module, a keyboard control module, communication module, buffer measurement modules. Among them, the buffer measurement module includes buffer chamber, measuring sensors inside the buffer chamber. 
The conductivity sensor uses a platinum black electrode cell constant is $1 \mathrm{~cm}^{-1}$, and its measurement range $2-20000 \mu \mathrm{S} / \mathrm{cm}$, the conductivity of the milk is usually in the range of $3000-7000 \mu \mathrm{S} / \mathrm{cm}$. Select conductance electrodes meet the measurement requirements. Because the conductivity electrode surface electroplating platinum black, increasing the surface area of the electrodes, the current density decreases. Meanwhile $100 \mathrm{~Hz}$ AC power as an incentive conductivity electrode, which can effectively reduce the polarization effects of the electrodes. Because the temperature is the main factor affecting the conductivity change, so integrated temperature sensor NTC10K in conductivity electrode, that resistance is at $25^{\circ} \mathrm{C} 10 \mathrm{~K} \mathrm{ohm}$ thermistor. Thermistor resistance decreases with increasing temperature, it is applied to compensate for temperature changes, its measurement range of $5^{\circ} \mathrm{C}$ to $50^{\circ} \mathrm{C}$. Because the conductivity measured temperature is standard temperature $25^{\circ}$ $\mathrm{C}$, so the temperature sensor options to meet the measurement requirements. Voltage analog signal will be output when the sensor data acquisition, signal processing circuit after it passes through the op amp and de-noising aspects of processing converted into a stable signal source. Then through the A/D converter converts the digital signal to achieve data collection.

Communication modules use MAX485 chip to complete TTL/RS485 level conversion, using the RS232/RS485 converter with the host institution to communications systems, achieve remote communications. RS485 communication system anti-interference ability, low impedance, transmission distance, to meet the communication needs. Single-chip microprocessor measuring unit collected data information is displayed on the LCD display easy for users observation data, confirm the work status of the device, through the communication module uploaded to the host computer for further processing, easy to implement host computer to the lower computer centralized management, greatly improving the usability of the system[10-11].

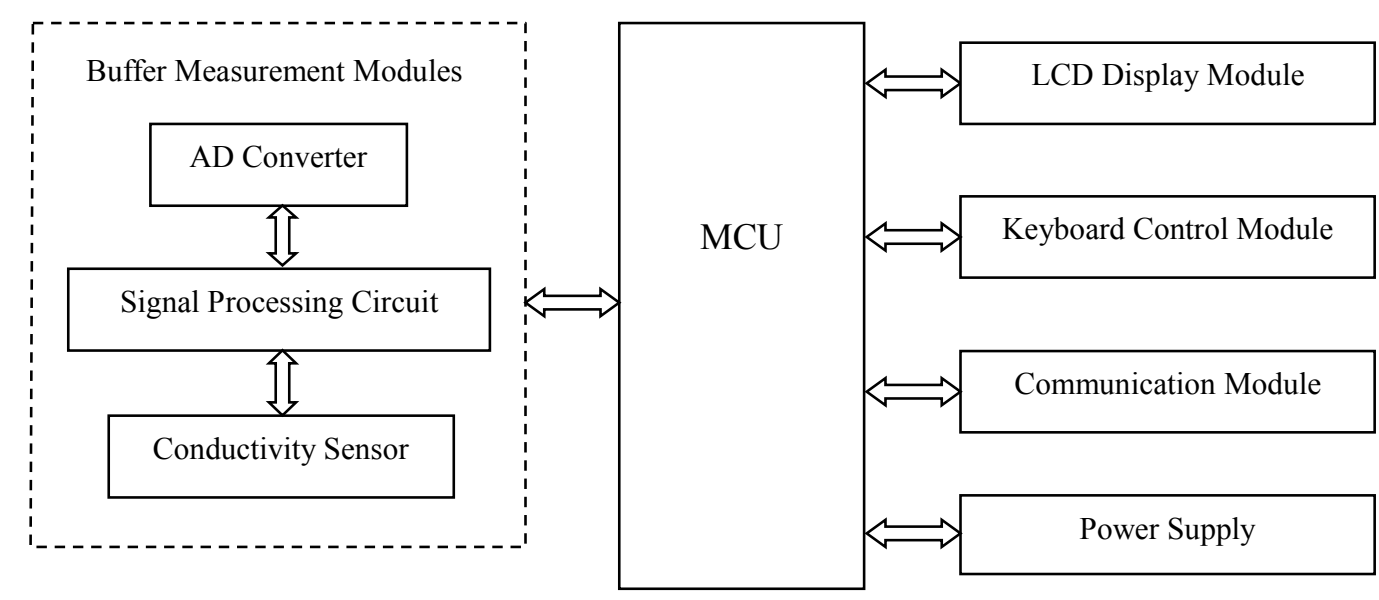

\section{Figure 2. Hardware Structure Diagram of the Measurement and Transmission Unit}

\subsection{Software Design of the System Description}

The main task of the monitoring system design is to achieve real-time monitoring of milk conductivity values. The main function of the software part is to realize the data processing and display, and to upload the data to the host computer servers and other functions. Figure 3 is a flowchart of the operation of the system. Measurement unit powered on, and then initialize each module. The system detects the sensor signal output later, after signal processing and A/D converted into a digital signal, the system will 
handle digital signals and use the function displayed on the LCD screen, while calling the data transfer unit will upload the data to the PC server. System has been set according to cows suffering from mastitis detection reference point for measuring the conductivity unit collected data to determine whether the output warning information. It is easy to real-time online monitoring of the health of the cows.

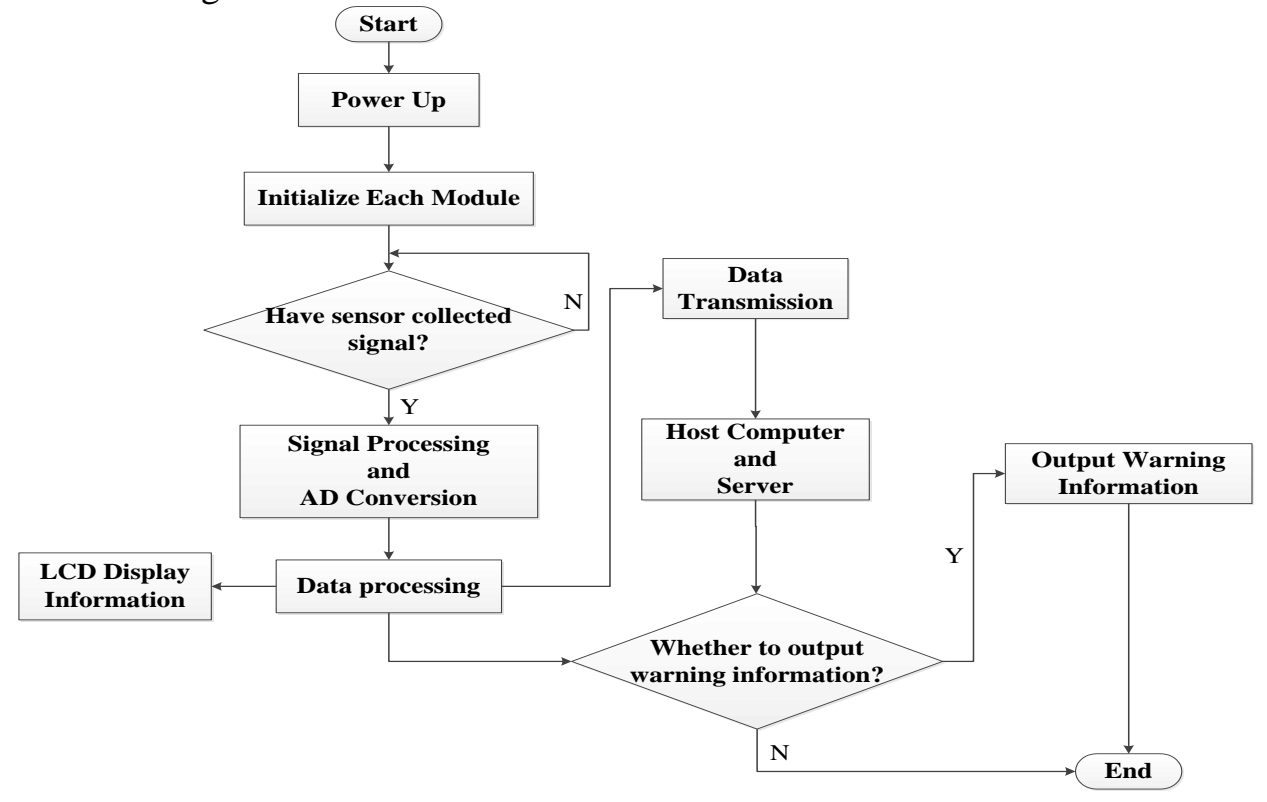

Figure 3. A Flow Diagram Design of the System

\subsubsection{Sensor Data Processing Method}

Figure 4 is a schematic diagram of the electrical conductivity measurements. According to the principle of conductivity measurement shows that the electrode and the test solutions constitute a conductivity cell, which can be used as resistance $R_{x} . U$ is a sinusoidal excitation voltage, $R_{f}$ is the feedback resistor and $U_{f}$ is the feedback voltage[12]. $G$ is the conductance of the test solution, as shown in the relationship between $G$ and $\mathrm{Rx}$ is shown in Equation (1):

$$
G=\frac{1}{R_{x}}(1)
$$

Using equation (1), we get the relationship between the feedback voltage $U_{f}$ and the conductance $G$, as (2) shown in Equation:

$$
G=\frac{U_{f}}{\left(U-U_{f}\right) \times R_{f}}(2) .
$$

The relationship between the conductivity and conductance of the solution, as shown in equation (3):

$$
K=Q \times G
$$

Where $K$ is the conductivity of the solution, $G$ is the conductance of the solution and $Q$ is the conductance cell constant.

Using equation (2) and (3), we get the relationship between the feedback voltage and the conductivity of the solution, as shown in equation (4):

$$
K=\frac{Q \times U_{f}}{\left(U-U_{f}\right) \times R_{f}}
$$


Where is the conductivity of the solution, $U_{f}$ is the feedback voltage. And because the parameter $Q, U, R_{f}$ are known, the feedback voltage can be obtained to calculate the conductivity of the solution.

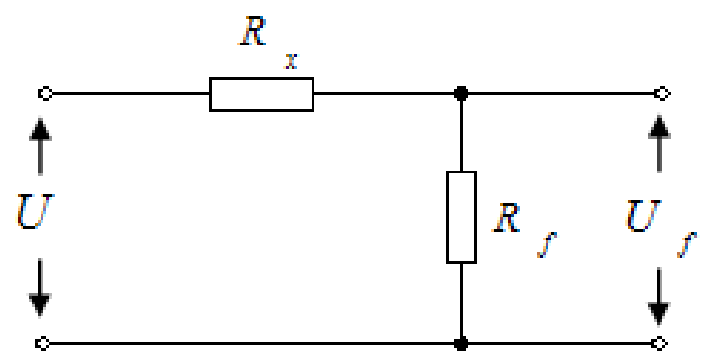

\section{Figure 4. A Schematic Diagram of the Electrical Conductivity Measurements}

\section{Simulation Analysis of the System}

\subsection{Conductivity and Feedback Voltage Relationship Verification}

According to the principle of conductivity measurement shows that a feedback resistor in series electrode used to obtain the corresponding feedback voltage. Because both ends of the electrode have a stable sinusoidal signal source, the feedback voltage from the change in conductivity of the solution will produce a weak change. Single-chip microprocessor processing the feedback voltage produced weak signal changes obtained corresponding solution conductivity value. Aiming at this, simulations were carried out to verify the conductivity and feedback voltage relationship[13-14]. Because the conductivity measured temperature is standard temperature $25^{\circ} \mathrm{C}$, so this experiment simulated the 120 group solution conductivity value at $25^{\circ} \mathrm{C}$, as much as possible so that it is evenly distributed measurement range of $0.7 \mathrm{mS} / \mathrm{cm}-11.7 \mathrm{mS} / \mathrm{cm}$ of inside, verification solution conductivity and the feedback voltage relationships through simulation circuit. Simulation results of the verification are shown in Figure 5.

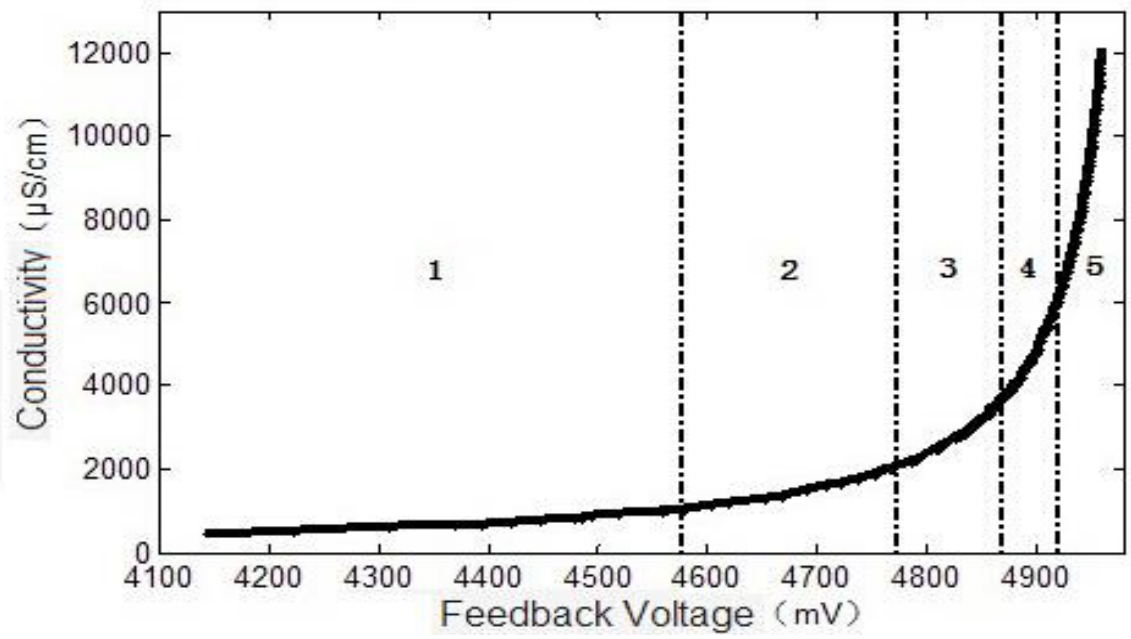

Figure 5. The Conductivity and Feedback Voltage Relationship Diagram

To meet the demand for conductivity measurement, the value of the feedback voltage is divided into five segments piecewise linear fitting, the fitting result as Equation (5) below. The linear regression coefficients $R^{2}$ were 0.97 or more, fitting better. Where $x$ 
is the feedback voltage $(\mathrm{mV}), f(x)_{25^{\circ} \mathrm{C}}$ is the conductivity values obtained by after the model processing $(\mu \mathrm{S} / \mathrm{cm})$.

$$
\begin{array}{lll}
f(x) 25^{\circ} \mathrm{C}=1.817 x-7266 & 4360 \leq x<4570 & R^{2}=0.9843 \\
f(x) 25^{\circ} \mathrm{C}=5.115 x-2.241 \mathrm{e}+004 & 4570 \leq x<4770 & R^{2}=0.9735 \\
f(x){ }_{25^{\circ} \mathrm{C}}=18.76 x-8.766 \mathrm{e}+004 & 4770 \leq x<4870 & R^{2}=0.9774 \\
f(x) 25^{\circ} \mathrm{C}=54.11 x-2.602 \mathrm{e}+005 & 4870 \leq x<4920 & R^{2}=0.9836 \\
f(x) 25^{\circ} \mathrm{C}=162.2 x-7.926 \mathrm{e}+005 & 4920 \leq x<4970 & R^{2}=0.975
\end{array}
$$

\subsection{Validation of Linear Fitting Model}

In order to verify the accuracy of the model, we conducted a verification test of piecewise linear fitting model. This experiment simulated the 97 group conductivity calibration solution conductivity value at $25^{\circ} \mathrm{C}$, as much as possible so that it is evenly distributed within the measurement range of $0.7 \mathrm{mS} / \mathrm{cm}-11.7 \mathrm{mS} / \mathrm{cm}$ s. Piecewise linear model of the system processing simulation data get measured values and simulation data for comparison. Simulation results of the validation are shown in Fig6.

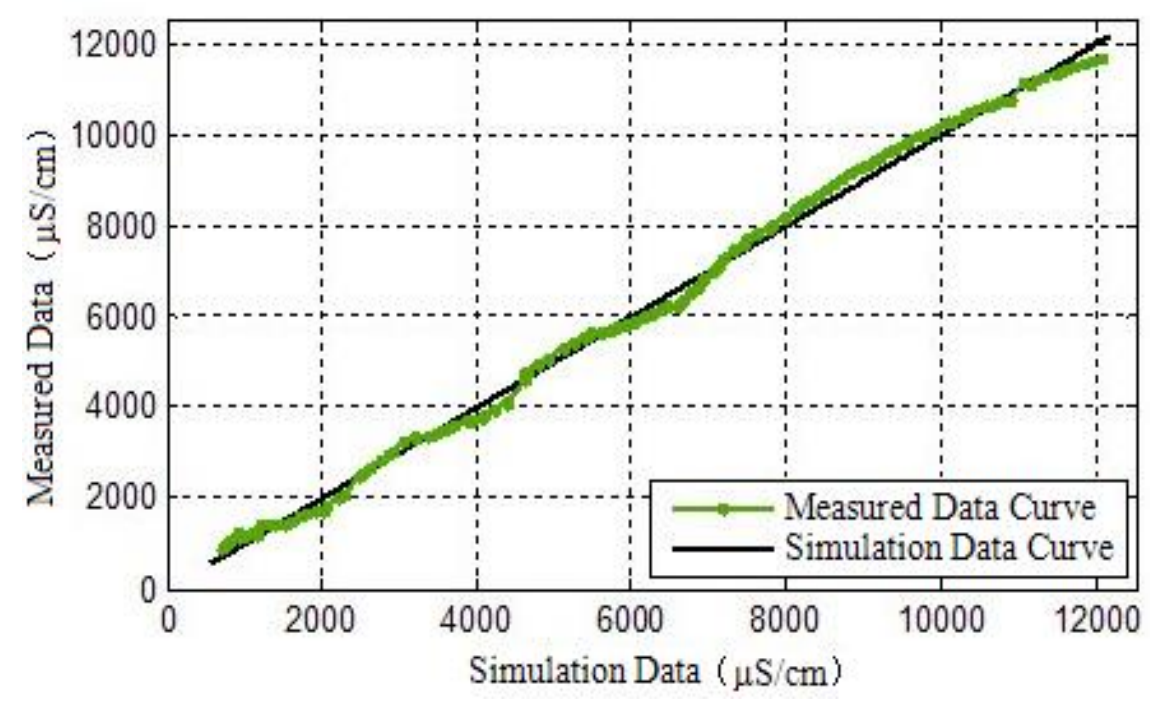

Figure 6. Simulation Verification Results of the Linear Model

Figure analysis shows that the measured data curve obtained by the piecewise linear model provide a better fit to the simulation data, linear fitting coefficient $R^{2}$ of measured data is 0.9876 , higher measurement accuracy. Meanwhile, in order to verify the accuracy of the measured data, we focused on the measurement data and the simulation data in accordance with equation (6) for the relative error calculation. According to the order of piecewise linear model to calculate the average relative error, the results were $1.37 \%$, $2.86 \%, 2.47 \%, 1.56 \%, 3.25 \%$. The average relative error of each segment is less than $4.0 \%$, calculated results show that the higher the conductivity measurement accuracy.

$$
\delta=\frac{\left|Y_{M}-Y_{S}\right|}{Y_{S}} \times 100 \%
$$

In the formula, $\delta$ is the relative error of the measurement, $Y_{M}$ is the measured conductivity value, $Y_{S}$ is the conductivity value of the simulation. 


\subsection{Validation of the Power Function Model}

In order to find other suitable model for the relationship between the conductivity and the feedback voltage, we implemented a power function curve fitting for the simulation data[15-16].Power function curve fits better, with $R^{2}$ fit coefficient of 0.9656. Figure 7 shows the two models validation of the electrical conductivity and the feedback voltage relationship. They are power function curve fitting models and piecewise linear curve fitting model.

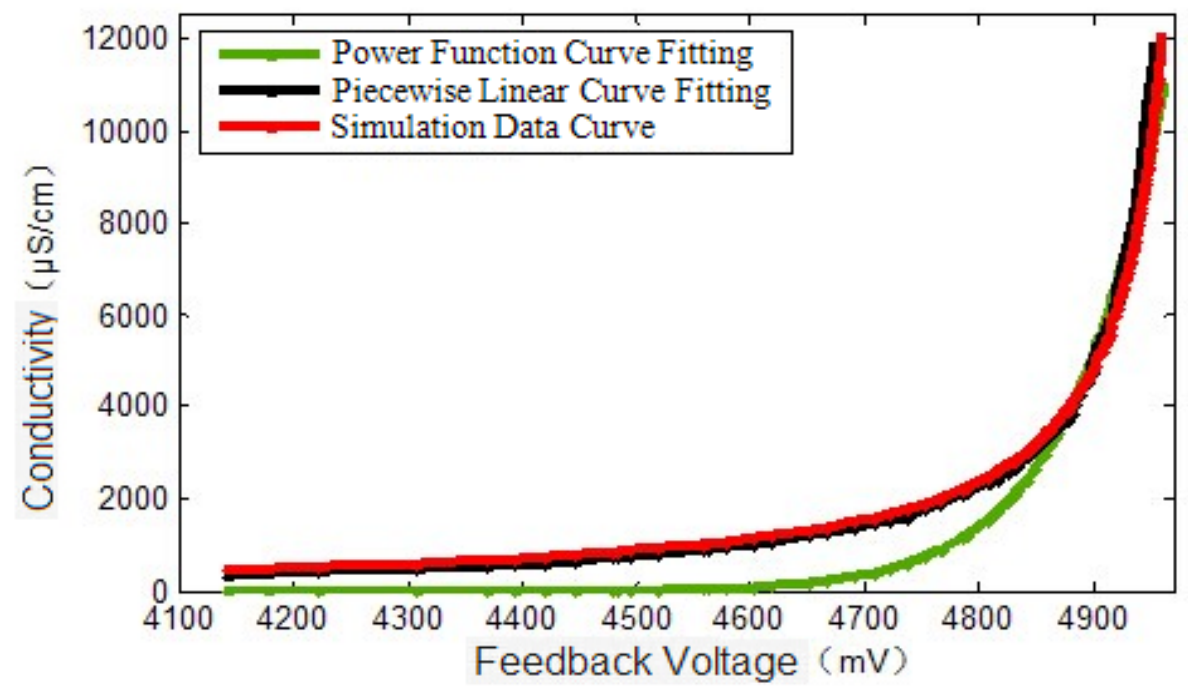

Figure 7. Validation Effect Drawing of Model

In order to verify the accuracy of the model, we performed a test to verify a power function curve fitting model. Verify the effect of power function curve model is shown in Figure 8.

Although the power function curve fitting coefficient $\mathrm{R}$ model is higher, but the actual measurement data and simulation data fitting results are poor. Power function curve model with piecewise linear model comparison showed that the measurement error of power function curve model is larger. After comparison shows that piecewise linear fitting model satisfies conductivity measurement system requirements, its relative error is smaller. By measuring the data and simulation data comparison shows that the measurement results can be accurate to $0.01 \mathrm{~ms} / \mathrm{cm}$, the measurement accuracy to meet the requirements.

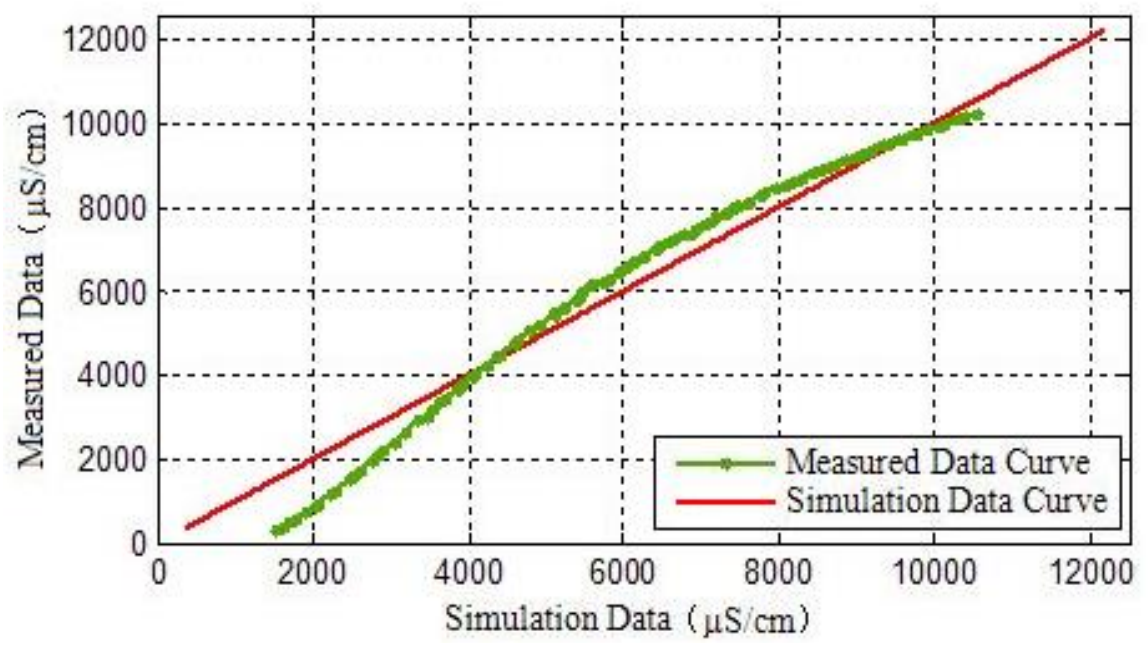

Figure 8. Simulation Verification Results of the Power Function Curve Model 


\section{Conclusion}

(1)Developed a milk conductivity real-time on-line monitoring system, the system consists of several parts of the measurement, transmission and service side, etc., with information collected milk conductivity, LCD display shows information, keyboard, data transmission and other functions.

(2) For the system, we conducted simulation experiments and analysis. For the relationship between electrical conductivity and the feedback voltage, we conducted simulation experiments. For piecewise linear fitting model and power function curve fitting models, as well as the accuracy of the model, we conducted a comparative simulation experiments. By comparison, we choose piecewise linear approximation model, which fits coefficients were higher than 0.97 , the measurement results can be accurate to $0.01 \mathrm{~ms} / \mathrm{cm}$, measuring range at $0.7 \mathrm{mS} / \mathrm{cm}-11.7 \mathrm{mS} / \mathrm{cm}$, better meet the conductivity measurement requirements.

(3)The reason of error during analysis of the power function curve fitting model may be because the change in the voltage value of the feedback amplitude is small. After the computation of operational amplifier, A/D converter, and the calculation formula, the error will be enlarged. For the selection of the model study will help to further improve the accuracy of the conductivity measurement.

(4) Milk conductivity value for real-time monitoring, prevention of cow mastitis or other diseases has an important role. However, due to the different regions, different types of cows and other reasons, the conductivity value of cow milk will be different. According to the actual application environment, the system can be set to cows suffering from mastitis detection reference point for real-time monitoring of breast health of dairy cows.

\section{Acknowledgements}

This work was supported in part by the National High-tech R\&D Program of China (863 Program)(2012AA101905), Key Technologies R\&D Program of Harbin(2014AB1BN035) and Post-doctoral research starting capital of Heilongjiang province(LBH-Q12146).

\section{References}

[1] C. Viguier, S. Arora, N. Gilmartin, K. Welbeck and R. O'Kennedy, "Trends in Biotechnology", vol. 27, no. $8,(2009)$.

[2] C. M. Jiang, X. M. Zhou, G. S. Zhou and Z. S. Wang, "China Animal Husbandry \& Veterinary Medicine", vol. 31, no. 5, (2004).

[3] C. J. Cui, S. P. Gu and Y. M. Zuo, "Transactions of the Chinese Society for Agricultural Machinery", vol. 42, no. 1,(2011).

[4] L. Yi, Y. P. Jin, W. Shi and J. W. Huang, “Animal Husbandry \& Veterinary Medicine”, vol. 38, no. 10, (2006).

[5] C. J. Cui, Z. K. Chen, X. C. Zhang, Y. M. Zuo, China Dairy Industry, vol. 41, no. 7, (2013).

[6] Q. Guo, L. Wang, W. Zhu and B. K. He, Guizhou Journal of Animal Husbandry and Veterinary Medicine, vol. 3, no. 5, (2009).

[7] E. Norberg, H. Hogeveen, I. R. Korsgaard, N. C. Friggens, K. H. M. N. Sloth and P. Løvendahl, Journal of Dairy Science, vol. 87, no. 4, (2004).

[8] M. Janzekovic, M. Brus, B. Mursec, P. Vinis, D. Stajnko and F. Cus, Journal of Achievements in Materials and Manufacturing Engineering, vol. 34, no. 1, (2009).

[9] J. Y. Li, F. Y. Tian and F. Li, Chinese Journal of Scientific Instrument, vol. 28, no. 2, (2007)

[10] Z. P. Chen and J. L. Du, Journal of Tianjin University of Technology, vol. 25, no. 2, (2009).

[11] G. P. Ling and J. X. Zhou, Chinese Journal of Scientific Instrument, vol. 26, no. 8, (2005).

[12] X. P. Chen and H. X. Chen, Chinese Journal of Scientific Instrument, vol. 27, no. 5, (2006).

[13] Y. H. Li, M. Z. Li, X. L. Deng, H. Sun and L. H. Zheng, Transactions of the Chinese Society of Agricultural Engineering, vol. 29, no. 9, (2013).

[14] F. H. Wang, Z. J. Qiu and D. F. Jie, "Transactions of the Chinese Society for Agricultural Machinery, vol. 40, no. 6, (2009). 
[15] W. Zhou, J. D. Bao and L. H. Ding, Journal of Test and Measurement Technology, vol. 27, no. 4, (2013).

[16] Q. Z. Wen and Y. Dai, Process Automation Instrumentation, vol. 30, no. 6, (2009).

\section{Authors}

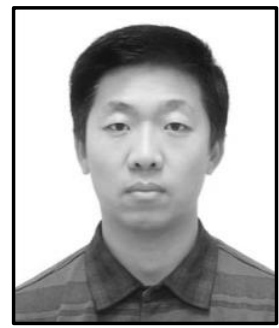

Weizheng Shen, (1977-), male, Ph.D., professor, mainly engaged in the research and application of information technology in agriculture. 
International Journal of Smart Home

Vol. 9, No. 5 (2015) 